WellBeing International

WBI Studies Repository

$12-2000$

\title{
Spontaneous Discrimination of Natural Stimuli by Chimpanzees (Pan troglodytes)
}

\author{
David A. Brown \\ Ohio State University \\ Sarah T. Boysen \\ Ohio State University
}

Follow this and additional works at: https://www.wellbeingintlstudiesrepository.org/acwp_asie

Part of the Animal Studies Commons, Comparative Psychology Commons, and the Other Animal

Sciences Commons

\section{Recommended Citation}

Brown, D. A., \& Boysen, S. T. (2000). Spontaneous discrimination of natural stimuli by chimpanzees (Pan troglodytes). Journal of Comparative Psychology, 114(4), 392.

This material is brought to you for free and open access by WellBeing International. It has been accepted for inclusion by an authorized administrator of the WBI Studies Repository. For more information, please contact wbisr-info@wellbeingintl.org.

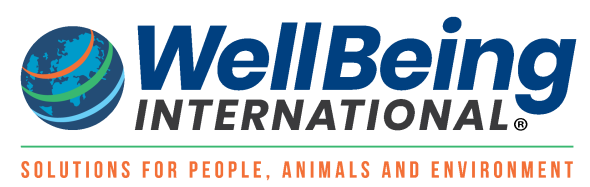




\title{
Spontaneous Discrimination of Natural Stimuli by Chimpanzees (Pan troglodytes)
}

\author{
David A. Brown ${ }^{1}$ and Sarah T. Boysen ${ }^{1,2}$ \\ ${ }^{1}$ Ohio State University \\ ${ }^{2}$ Emory University
}

\begin{abstract}
$\underline{\text { ABSTRACT }}$
Six chimpanzees (Pan troglodytes) were presented with pairs of color photographic images of 5 different categories of animals (cat, chimp, gorilla, tiger, fish). The subjects responded to each pair using symbols for "same" and "different." Both within- and between-category discriminations were tested, and all chimpanzees classified the image pairs in accordance with the 5 experimenter-defined categories under conditions of nondifferential reinforcement. Although previous studies have demonstrated identification or discrimination of natural categories by nonhuman animals, subjects were typically differentially reinforced for their responses. The present findings demonstrate that chimpanzees can classify natural objects spontaneously and that such classifications may be similar to those that would be observed in human subjects.
\end{abstract}

Natural categories are open-ended and are formed through interactions with the environment (Herrnstein \& de Villiers, 1980; Hermstein, Loveland, \& Cable, 1976). Therefore, research on category discrimination using natural categories may better approximate discrimination of stimuli under natural circumstances than if simpler, more controlled stimuli are used (Malt, 1995). Interest in the discrimination of natural categories was prompted by the demonstration that pigeons could discriminate slides containing humans from others not containing humans (Herrnstein \& Loveland, 1964). Numerous studies with several species of animals followed; researchers conducting these studies investigated classification of stimuli from a variety of natural categories, including fish (Hermstein \& de Villiers, 1980); trees, bodies of water (Herrnstein et al., 1976); people (D'Amato \& Van Sant, 1988; Hermstein \& Loveland, 1964; Schrier \& Brady, 1987); animals, food (Fabre-Thorpe, Richard, \& Thorpe, 1998); and various species of primates (Me \& Markowitsch, 1987; Phillips, 1996; Sands, Lincoln, \& Wright, 1982; Yoshikubo, 1985). Natural categories do not necessarily contain "natural" objects. They may, instead, contain objects with which an animal has had no natural experience. For instance, Herrnstein and de Villiers (1980) reported classification of photographs of fish by pigeons, although pigeons would not normally encounter fish in their natural habitat. Animals have been shown to discriminate equally well between categories of natural objects and those composed of man-made objects (Bhatt, Wasserman, Reynolds, \& Knauss, 1988; Wasserman, Kiedinger, \& Bhatt, 1988), although Herrnstein (1985) noted that pigeons did not perform as well with man-made objects when the categories were based on function. However, from a strictly perceptual perspective, natural categories were more easily discriminated than simpler stimuli such as monochromatic lights, presumably because some species' perceptual systems are naturally prepared to process three-dimensional, complex objects (Herrnstein \& de Villiers, 1980). 
Discrimination of natural categories has also been studied in human infants (Eimas \& Quinn, 1994; Mandler \& McDonough, 1993; Oakes, Coppage, \& Dingel, 1997; Quinn, Eimas, \& Rosenkrantz, 1993). A habituation-dishabituation approach has often been used in such studies (e.g., Eimas \& Quinn, 1994; Quinn et al., 1993). Alternatively, some researchers have examined the manner in which infants manipulate stimuli from different categories (Mandler \& McDonough, 1993; Oakes et al., 1997), and this latter method has also been utilized with young chimpanzees (Spinozzi, 1993). Although such tasks may be capable of demonstrating how animals discriminate between stimuli, they may not necessarily demonstrate how animals identify categories.

Hayes and Nissen (1971) reported discrimination between pairs of drawings of natural objects by a 5year-old, home-reared chimpanzee (Viki). Her performance was comparable to that of children of approximately the same age. Explanations by the children as to how they made their choices suggested that they may not have used the same categories used by the experimenters in forming the pairs. Similarly, it has been suggested that the strategies used by nonhuman animals to discriminate natural categories may be different than those used by the experimenters defining the categories (Herrnstein, 1985; Schrier \& Brady, 1987). For example, D'Amato and Van Sant (1988) reported successful discrimination between slides with and without humans by capuchin monkeys (Cebus apella). However, errors made by the monkeys suggested that some responses were elicited by discrete features not relevant to the category of "human," thus supporting the notion that nonhuman animals may classify natural objects differently than human subjects do. Thompson (1995) offered a more thorough review of this issue, with a similar conclusion. Alternatively, Fabre-Thorpe et al. (1998) have suggested that differences in performance between humans and monkeys may be due to a propensity for the monkeys to respond more quickly, thereby reducing the time spent evaluating the stimuli and consequently increasing the number of errors.

One may wonder, therefore, whether nonhuman animals subjectively discriminate among natural categories in a manner similar to humans, or whether the results of such research merely indicate that the animals have learned the categories during experimental training. In two studies, researchers have attempted to address this question by omitting explicit training of the categories. Under such conditions, Me and Markowitsch (1987) found that squirrel monkeys and humans classified stimuli from a variety of categories in a similar manner. Although each trial was novel, the monkeys were differentially reinforced, and, therefore, still could have learned the categories during testing. Sands et al. (1982) offered perhaps the most impressive evidence of subjective discrimination/categorization by rhesus macaques (Macaco mulatto). They found that macaques occasionally judged humans and monkeys, as well as trees and flowers, as the same, despite differential reinforcement for discriminating between these categories. Although suggestive, the data did not necessarily reveal categorization, but rather relative similarity judgments.

Although current evidence strongly suggests that chimpanzees may utilize relational concepts without specific training (see Matsuzawa, 1990; Tanaka, 1995), nonhuman animals have not yet been shown to explicitly discriminate among natural categories entirely without differential reinforcement. Thus the question of whether such discriminations might emerge spontaneously remains unanswered. The present study was conducted to evaluate the potential for subjective classification of natural categories by chimpanzees, without explicit training with differential reinforcement. The chimpanzees were presented with pairs of images, each depicting a single animal, and were required to judge each pair as "same" or "different" (cf. Premack, 1983). A number of studies have shown that both chimpanzees and macaques can make reliable same-different judgments (e.g., Fujita, 1983; Neiworth \& Wright, 1994; Oden, Thompson, \& Premack, 1990; Sands et al., 1982). For instance, Neiworth and Wright demonstrated that rhesus macaques judged pairs of pictures depicting different views of the same object to be the same. 
The results were interpreted as indicative of categorical discriminations, even though each category comprised a single object (albeit varying views of that object). In the present study, however, stimulus pairs depicted either two different stimuli from the same category or two stimuli from different categories. Thus, comparisons of stimuli both within and between categories were presented to determine whether chimpanzees would discriminate among natural categories without explicit training.

\section{Experiment 1}

Because in previous studies on category discrimination with nonhuman animals, researchers typically have attempted to train animals to discriminate between experimenter-defined categories, little evidence exists for subjective discrimination of natural stimuli. In the present experiment, we explored the ability of chimpanzees to judge images from five different categories of animals as the same or different, with no reinforcement contingencies to guide their judgments.

\section{Method}

Subjects. Subjects were 6 chimpanzees (Pan troglodytes), including 4 adult males (9 to 18 years old) and 2 adult females (16 and 39 years old). Chimpanzees were housed in social groups with 24-hr indooroutdoor access and were only separated from conspecifics for individual testing. Five of the 6 chimpanzees were experimentally sophisticated, having participated in numerous cognitive studies (e.g., Boysen, 1997; Boysen, Mukobi, \& Berntson, 1999; Thompson, Oden, \& Boysen, 1997). Furthermore, they had been previously trained to use symbols representing the concepts of same and different and had recently used them to make reliable same-different judgments between Arabic numerals and dot arrays (Himes, 1999). Performance on one of these recent experiments is depicted in Figure 1, demonstrating competent use of the same and different symbols. The youngest chimpanzee was relatively experimentally naive. He had been trained to make responses to visual stimuli on a cathode-ray tube (CRT) monitor interfaced with a touch-frame system and had begun to learn the use of the numerals 1,2, 3 , and 4. However, because he had no prior training with the same-different symbols, his participation in the present study served as a control.

Apparatus. Chimpanzees were tested individually in a room that could be accessed directly from their outdoor play areas. They sat in front of a 14-inch video graphics array (VGA) computer monitor mounted with a Carroll Touch-Frame (Round Rock, TX). Stimuli were presented on the monitor, and the touchframe system recorded responses as chimpanzees indicated their choices via the touch-frame. The experimenter was seated on the other side of a window and controlled the presentation of stimuli through an IBM 80486 computer.

Stimuli. Test stimuli consisted of 35 digitized color images $(240 \times 240$ pixels; approximately $8.5 \times 8.5 \mathrm{~cm})$ of five types of animals. Each image represented a single, unique individual. To minimize extraneous cues, we portrayed each animal on the same light blue background. The overall size of each stimulus, including the background, was the same, but the relative size of the animals within the images was somewhat variable and was not related to the animals' true sizes. The largest image covered $56 \%$ of the background, and the smallest covered $16 \%$ of the background; the animals also varied in body posture and orientation. To further attenuate potentially confounding characteristics of the stimuli (i.e., to minimize age-related perceptual cues), we used only images of adult animals. In addition, only photographs of animals with neutral facial expressions and body postures were used, with visibility of teeth minimal, to control for potentially aversive or attractive images. All images displayed all or most of the body and at least part of the face and were randomly chosen from a pool of 116 animal photographs, available as digitized images. 
Figure 1. Performance on a previous same-different task by the 5 experienced chimpanzees in the present study. The same-different symbols described for Experiment 1 were used to make same-different judgments regarding pairs of Arabic numerals and dot arrays. The data depicted represent responses to novel stimulus presentations. From A Chronometric Analysis of Same/Different Judgments Based on Quantity by Chimpanzees (Pan troglodytes), Unpublished master's thesis (p. 84) by G. T. Himes, 1999, Ohio State University, Columbus.

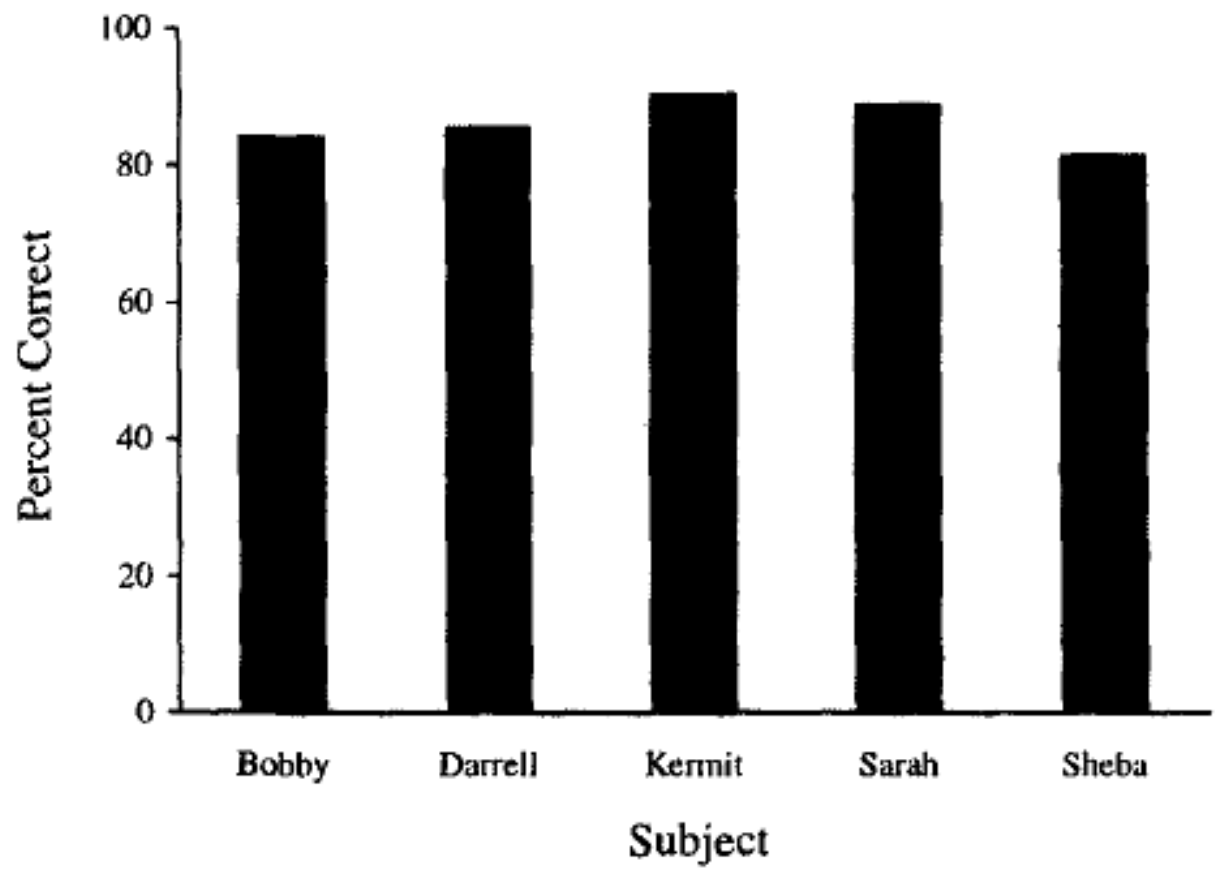

Five categories of animals were presented: chimpanzees, gorillas, house cats, tigers, and fish, with seven different exemplars in each category. Chimpanzee images were used because we hypothesized that this category would be of interest to the subjects, and gorilla images were used because of their perceptual similarity to chimpanzees. Likewise, cats and tigers are perceptually similar to one another, but more distinct from apes. The remaining category, fish, was the most perceptually distinct from all other categories.

The response symbols representing same and different were both black rectangles measuring $199 \times 142$ pixels (approximately $7 \times 5 \mathrm{~cm}$ ) surrounded by white borders. The same symbol had a white heart shape in the center of the rectangle, and the different symbol was a diagonal white stripe, measuring approximately $2 \mathrm{~cm}$ in width, from the upper right corner to the lower left corner of the rectangle. Figure 2 presents a typical example of test stimuli that were displayed on the computer screen during a single trial. Although the stimuli in Figure 2 are depicted in black and white, all stimuli were presented in color during testing.

Procedure. Each session consisted of 15 trials, with chimpanzees completing one to three sessions daily, 5 days a week, for a total of 315 trials, all novel. A trial consisted of two different test stimuli presented simultaneously on the upper half of the screen, with the two response symbols for same and different in the lower left and right corners of the screen, respectively. The background for the upper portion of the screen was white, and the background for the lower portion of the screen was black, to better separate the test stimuli from the response symbols. 
Figure 2. Example of a cat-tiger comparison, with the same and different response symbols in the lower left and right corners, respectively.

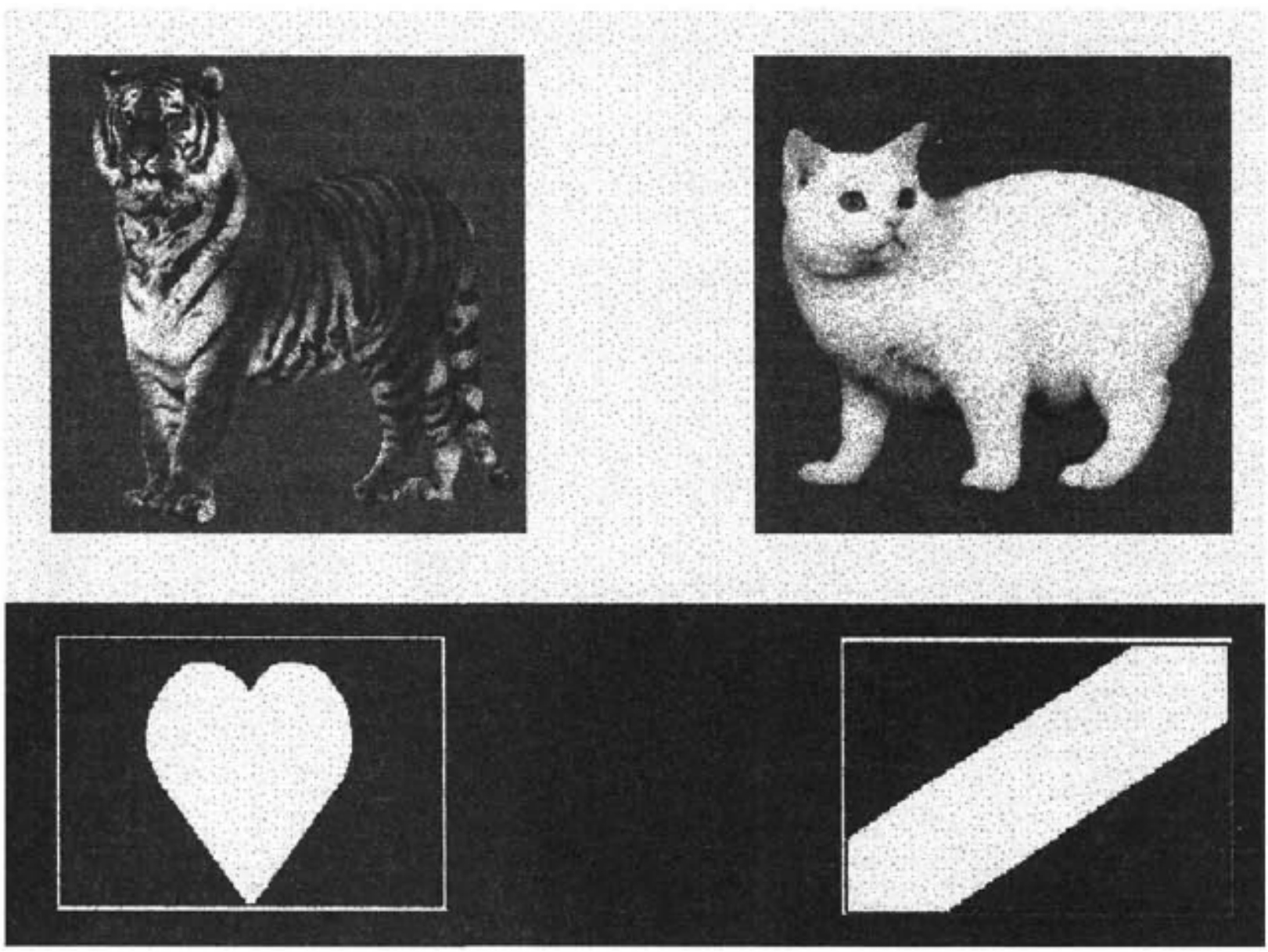

The experimenter initiated each trial by pressing a key on the keyboard, and the test stimuli and response symbols immediately appeared on the screen. Consequently, intertrial intervals were not consistent from trial to trial, but were typically 3 to $4 \mathrm{~s}$. A trial ended when the chimpanzee touched one of the response symbols (same or different). Following a response, the screen went blank, and the experimenter provided nondifferential reinforcement, including verbal praise and a small candy for every trial, regardless of the chimpanzee's response. Stimulus pairs were presented in quasirandom order, with no more than three consecutive within-category or cross-category comparisons. Left-right screen position of exemplars from each category was counterbalanced within a session. Two to six exemplars from each category were presented during a session. However, on average, four different exemplars from each category were presented per session, and two images typically appeared twice within a session, but always in a different pairing. A total of 315 trials included 21 within-category comparisons for each category, and each category was also compared to each of the other categories 21 times, for a total of 105 within-category comparisons and 210 cross-category comparisons.

Data analysis. An alpha level of .05 was used for all statistical tests. Correct categorical responses were defined as same responses to within-category comparisons, and different responses to cross-category comparisons. If subjects had responded randomly, with equal proportions of same and different responses, chance would have been .50. Binomial tests showed that performance for each subject was significantly different than chance, $p<.05, N=315$. A sign test found overall performance across all 
subjects $(N=5)$ to be significantly greater than chance, $p<.05$. However, because there were an unequal number of same and different trials, a bias toward one response key could have artificially raised or lowered categorical response levels. Twice as many cross-category comparisons (e.g., cat vs. fish) were presented as within-category comparisons (e.g., cat vs. cat). Therefore, a bias for responding different, for example, would improve performance simply because there were more different than same trials. As a result, the control subject, Digger, responded categorically at a level significantly below chance. Hypothetically, his responses should have been at chance, because he was not trained on the representational nature of the response symbols. However, because he showed a position preference when responding (60\% left; $40 \%$ right), his overall performance was differentially affected by this spatial bias.

Table 1. Proportion of Same and Different Responses in Experiment 1 and Chance Level of Categorical Responding, With Correction for Response Bias

\begin{tabular}{|lcccc|}
\hline & & \multicolumn{2}{c|}{ Proportion of responses } & \\
\cline { 2 - 4 } Subject & Age/sex & Same & Different & Chance level of categorization \\
\hline Bobby & $11 / \mathrm{M}$ & .30 & .70 & .57 \\
Darrell & $18 / \mathrm{M}$ & .32 & .68 & .56 \\
Kermit & $18 / \mathrm{M}$ & .53 & .47 & .49 \\
Sarah & $39 / \mathrm{F}$ & .58 & .42 & .47 \\
Sheba & $16 / \mathrm{F}$ & .41 & .59 & .53 \\
Digger $^{\mathrm{a}}$ & $9 / \mathrm{M}$ & .60 & .40 & .47 \\
Overall $^{\mathrm{b}}$ & & .43 & .57 & .52 \\
\hline
\end{tabular}

Note. $\mathrm{M}=$ male; $\mathrm{F}=$ female.

${ }^{a}$ Control subject.

${ }^{\mathrm{b}}$ Calculated using data from all subjects except Digger.

To account for possible response biases, we calculated modified chance levels on the basis of the observed distribution of same and different responses. The following equation was used to calculate chance levels and to account for any positional biases: Chance $=(S T) \times(S R)+(D T) \times(D R)$, where ST is the proportion of same trials, and SR is the proportion of same responses; DT is the proportion of different trials, and DR is the proportion of different responses.

Despite possible biases, however, chance responding assumes that same and different responses were equally distributed among the same and different trials. Across all subjects (except the control) and all trials, the proportion of same trials was .33, and different trials .67. However, the proportion of same responses was .43 , and different responses .57 . Thus, using the above equation, we find that the overall chance level of performance was .52. The proportion of same and different responses and modified chance levels of categorical responding are shown in Table 1. Using the control subject's modified chance level of .47 within a binomial test, we found his responses to be no different from chance. Therefore, all subsequent statistical tests used corrected chance levels calculated using the above equation.

\section{Results}

The control subject, Digger, performed at chance with respect to all five categories. However, the other 5 chimpanzees responded categorically on $69 \%$ of all trials (chance $=52 \%$; see Table 1 ), and each subject showed the same general pattern of responding to the stimuli. The contrast between Digger's responses 
and those of the other 5 chimpanzees supports the claim that the same and different symbols elicited meaningful responses with respect to the test stimuli from these 5 subjects. Digger's data were excluded from all further analyses.

Twenty-one trials were completed for each type of comparison (e.g., tiger-tiger, chimp-gorilla). To examine changes in responding during testing, we divided the 21 trials into three blocks of 7 trials. McNemar change tests (Siegel \& Castellan, 1988) were used to compare responses during the first and last block of trials for each subject, for each type of comparison. No differences were observed between the different trial blocks ( $p>.05, N=7$ ), thus demonstrating that categorical responding was present during both early and later trials and was not likely acquired during testing.

Table 2. Percentage of Categorical Responses in Experiment 1 Relative to Individual Chance Levels, With Correction for Bias, Overall and for Each Type of Comparison by Subject

\begin{tabular}{|c|c|c|c|c|c|c|}
\hline \multirow[b]{2}{*}{ Comparison } & \multicolumn{6}{|c|}{ Subject } \\
\hline & Bobby & Darrell & Kermit & Sarah & Sheba & Digger $^{\mathrm{a}}$ \\
\hline Cat-cat & $+30 * *$ & $+20 * \star$ & $+42^{* \star}$ & +18 & $+40 * *$ & +7 \\
\hline Chimp-chimp & +3 & +6 & $+37^{\star \star}$ & +18 & +16 & -3 \\
\hline Fish-fish & $+40^{\star \star}$ & +16 & $+47^{\star \star}$ & $+23^{\star \star}$ & $+40^{\star \star}$ & -12 \\
\hline Gorilla-gorilla & +18 & +6 & $+37^{\star \star}$ & $+32^{\star \star}$ & $+30^{\star *}$ & -17 \\
\hline Tiger-tiger & +13 & +11 & $+37^{\star \star}$ & $+23^{\star \star}$ & $+35^{\star \star}$ & +2 \\
\hline Cat-chimp & +16 & +8 & $+33^{\star \star}$ & -4 & +12 & -2 \\
\hline Cat-fish & +16 & -6 & $+33^{\star \star}$ & $+20^{\star \star}$ & +17 & -2 \\
\hline Cat-gorilla & -3 & +8 & +10 & $+29^{\star \star}$ & +12 & -7 \\
\hline Cat-tiger & +1 & +13 & -14 & -13 & +3 & +17 \\
\hline Chimp-fish & +6 & +3 & $+20^{* \star}$ & +9 & +12 & -11 \\
\hline Chimp-gorilla & -8 & +3 & +1 & $+25^{\star \star}$ & +17 & -16 \\
\hline Chimp-tiger & $+20^{\star \star}$ & +8 & $+38^{\star \star}$ & +10 & +17 & -7 \\
\hline Fish-gorilla & $+20^{\star \star}$ & +8 & $+33^{\star \star}$ & $+20^{\star \star}$ & $+22^{\star \star}$ & +3 \\
\hline Fish-tiger & $+20 * *$ & +18 & $+24^{\star \star}$ & +6 & $+27^{\star \star}$ & +3 \\
\hline Gorilla-tiger & $+20^{\star *}$ & -6 & +15 & +10 & +17 & -7 \\
\hline Overall & $+14^{*}$ & $+8^{*}$ & $+27^{*}$ & $+15^{*}$ & $+21^{*}$ & -4 \\
\hline
\end{tabular}

Note. Plus (+) indicates above-chance categorization, and minus (-) indicates below-chance categorization.

Chance levels for each subject are reported in Table 1.

${ }^{a}$ Control subject.

${ }^{*} p \leq .05$ using binomial test, $N=315$.

${ }^{*} p \leq .05$ using binomial test, $N=21$.

The animals in the images were not all the same size, and it may have been possible for chimpanzees to use size as a decision criterion. Of the 315 comparisons, 75 paired images differed in size by $20 \%$ or more. Chi-square tests were used to determine if responses differed as a function of this size disparity; however, all analyses were nonsignificant for all chimpanzees. Another potentially influential variable was postural differences between images; fish comparisons were excluded from this analysis. Among the remaining four categories, animals were depicted standing, sitting, or lying down. However, chi-square tests revealed no significant effect of posture for any chimpanzee. Additional analyses were completed for image orientation (left, right, or straight ahead), and similarly, chi-square tests showed no significant effect of orientation for any chimpanzee. Thus, overall, same-different discriminations by the chimps were 
not significantly influenced by differences in size, posture, or orientation of the animals depicted in the stimuli.

Because every category of animal was compared against every other category, a total of 15 different comparisons were possible (i.e., cat-cat, cat-chimp, cat-fish, etc.). Overall, all 5 chimpanzees responded categorically more frequently than expected by chance (see Table 2), and a sign test indicated that this pattern of responding was statistically significant $(p<.05, N=5)$. The data further indicated that all chimpanzees responded categorically at greater than chance levels on each of the five within-category comparisons. Again, a sign test indicated that their performance was statistically significant $(p<.05, N=$ $5)$. The chimps also responded categorically at a significant level on 4 of the 10 cross-category comparisons $(p<.05, N=5)$. Four of the 5 chimpanzees responded categorically to 5 of the 6 remaining cross-category comparisons, although because of the small number of subjects, this finding did not reach significance with a sign test. Figure 3 depicts the overall proportion of same responses for each type of comparison; the data in Figure 3 are compared against the overall chance level reported in Table 1, calculated by pooling all chimpanzees' responses (minus the control subject).

All chimpanzees responded same more frequently to all within-category comparisons than they did to any cross-category comparisons, resulting in responses similar to those shown in Figure 3 for individual chimpanzees. Among the cross-category comparisons, the most same responses were elicited by cattiger comparisons. The chimpanzees responded same to cat-tiger images on $45 \%$ of the trials, suggesting some difficulty in discriminating these two categories. In contrast, subjects responded "same" on $26 \%$ of all other cross-category comparisons, and $68 \%$ of all within-category comparisons. See Table 2 for a summary of each chimpanzee's performance on the 15 comparisons; unlike in Figure 3, chance levels in Table 2 represent individual chance levels, as reported in Table 1.

\section{Discussion}

The results of Experiment 1 demonstrated that the chimpanzees' discriminations were generally in agreement with the five experimenter-defined categories, without explicit training. Under some conditions, therefore, chimpanzees may classify objects in their environment in much the same way as many adult humans do. Alternatively, the chimpanzees may have judged some stimuli to be the same simply because they could not perceptually discriminate between them. Experiment 2 was designed to test this hypothesis and to examine the discriminability of the stimuli.

\section{Experiment 2}

The conclusion that the chimpanzees made categorical discriminations in Experiment 1 rests on the assumption that they judged stimulus pairs as the same because of their perceptual similarity, not because the stimuli were perceived as identical (Zayan \& Vauclair, 1998). Thus, it was assumed that the chimpanzees judged certain stimuli to be the same despite perceived differences between them. To examine the validity of this assumption, we needed to demonstrate that the chimpanzees could discriminate between stimuli judged to be the same, even though the stimuli most often judged as same were potentially the least discriminable from one another. Stimulus pairs used in Experiment 2 consisted of those pairs unanimously judged to be the same in Experiment 1.

\section{Method}

Subjects and apparatus. The same subjects who participated in Experiment 1 were tested in Experiment 2 , with the exception of the control subject $(N=5)$. All data were collected via the touch-frame system described previously. 
Stimuli. Twelve stimulus pairs from Experiment 1 were judged to be the same by all 5 chimpanzees and were thus used as stimuli for the present experiment. These included three pairs of cats, one pair of chimps, four pairs of fish, one pair of gorillas, two pairs of tigers, and a chimp-gorilla pair. In this latter pair, the chimp and gorilla were each sitting, with one arm outstretched. In Experiment 1, the distinctive postural similarity of the two images may have served as a discriminative cue, resulting in them being judged as the same, despite the species difference. Each stimulus measured $230 \times 230$ pixels in size.

Figure 3. Proportion of same responses for each type of comparison. The chance level represents the overall proportion of same responses. Gray bars represent within-category comparisons, and black bars represent cross-category comparisons. *Responses are significantly different from chance; sign test, $\mathrm{p} \leq .05, \mathrm{~N}=5$.

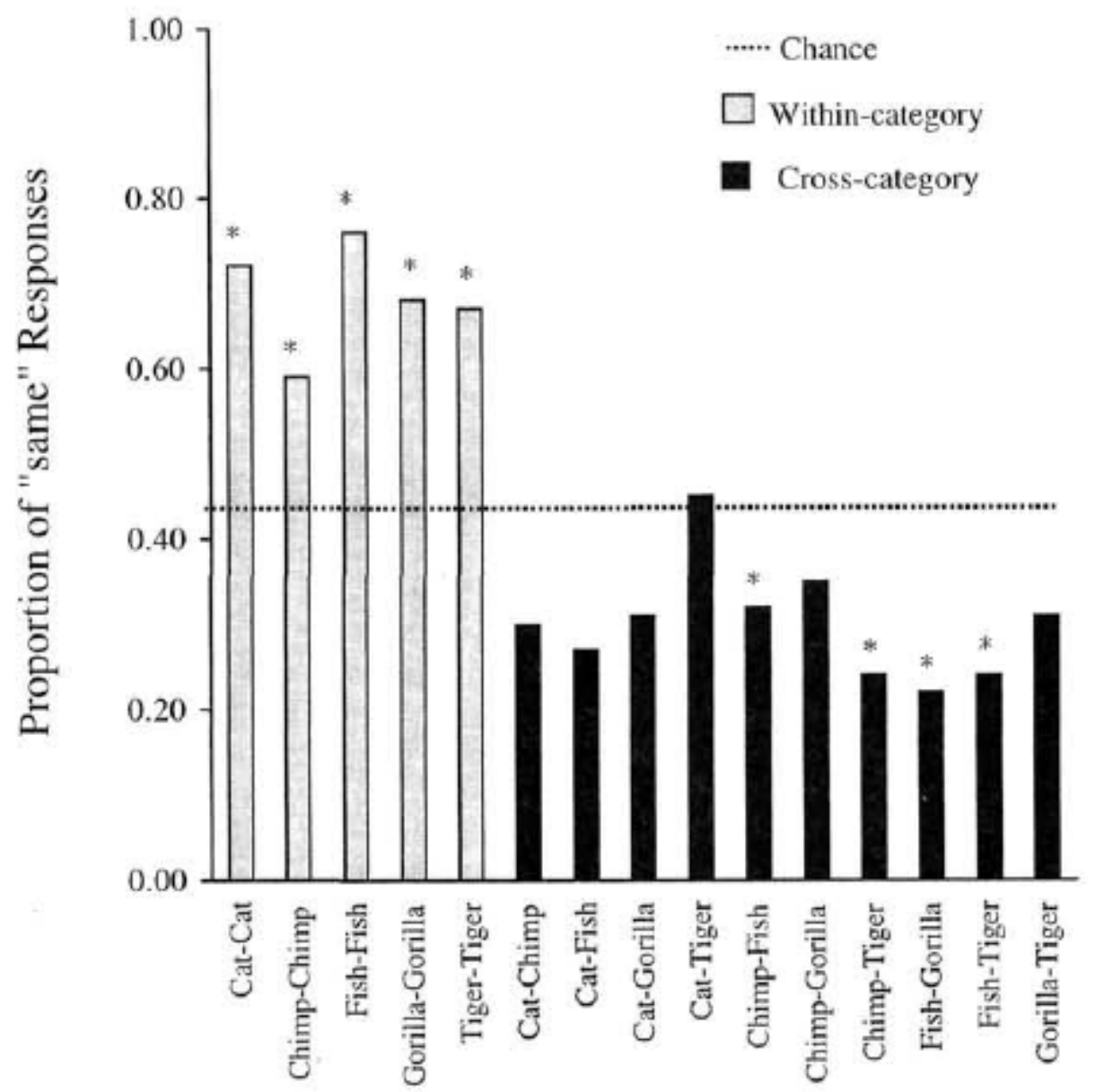

\section{Comparison Type}

Procedure. A simultaneous match-to-sample procedure was used. Each trial included a sample stimulus on a white background centered on the upper portion of the screen, with two comparison stimuli in the lower left and right corners on a black background, with one of the comparison stimuli identical to the sample. Each session consisted of 12 trials, with each stimulus pair presented once. Within each block of four sessions, each of the 12 stimuli was presented as the sample twice. Positions of the comparison stimuli were counterbalanced. Therefore, for each comparison pair, 4 unique trials were presented for a total of 48 unique trials. Trial order was randomly determined, with no more than three successive correct responses on the left or right, and no more than four alternating responses. Chimpanzees were reinforced 
only for choosing the matching comparison stimulus on each trial. Testing continued until each chimpanzee demonstrated a statistically significant level of performance (10 or more correct responses in 12 trials, $a=.05)$ for two consecutive sessions.

\section{Results and Discussion}

Across all subjects, the number of trials to criterion ranged from 156 to 276 . During the two criterion sessions, each chimpanzee performed better than expected by chance (.5), as determined with a binomial test, $p<.05, N=24$, with performance ranging from $83 \%$ to $96 \%$ correct. These results demonstrate that the chimpanzees were able to discriminate between the stimulus pairs. Thus, it is likely that the stimulus pairs that were previously judged to be the same in Experiment 1 were perceptually discriminable but were nevertheless judged categorically to be the same.

\section{General Discussion}

The most striking finding from Experiment 1 was that the chimpanzees spontaneously discriminated between stimuli from natural categories in much the same way humans might. The present results appear to meet the criteria for discrimination of open-ended categories, as described by Zayan and Vauclair (1998), in that (a) stimuli belonging to different categories were discriminated from one another, and (b) stimuli within a single category were discriminably different from one another. Several possible explanations could account for the chimps' performance. For example, the discriminations could have resulted entirely from stimulus generalization or from the mediating effects of fully-formed human-like concepts (Schrier \& Brady, 1987). However, there is currently no consensus among researchers as to how natural categories are formed, either for human or nonhuman animals (Herrnstein et al., 1976; Malt, 1995; Roberts \& Mazmanian, 1988).

Cerella (1979) suggested that pigeons may be prone to discriminate natural objects at a taxonomic level, in which some categories (e.g., oak leaf) may be more easily identified than subordinate categories (e.g., different kinds of oak leaves). Evidence in support of this hypothesis was demonstrated with pigeons and monkeys (Roberts \& Mazmanian, 1988), as well as humans (Mandler \& McDonough, 1993). Roberts and Mazmanian presented pigeons, squirrel monkeys (Saimiri sciureus), and adult humans with three different discrimination tasks, including kingfishers-other birds, birds-other animals, and animalsnonanimals. The human subjects found it most difficult to learn the most concrete (kingfishers vs. birds) discrimination, whereas squirrel monkeys and pigeons learned the most global and concrete categories but were unable to effectively perform the intermediate discrimination (birds vs. other animals). Thus, categories can be represented at different levels of abstraction. For example, a kingfisher may also be referred to as a bird or an animal, both of which are equally accurate classifications. However, one category is typically preferred over others. This preferred level of abstraction is often referred to as a basic-level category (Mervis \& Rosch, 1981). Medin and Smith (1984) described the basic level as "that which maximizes the number of distinctive properties, where a distinctive property is common to most members of a concept but lacking to most members of contrasting concepts" (p. 124). Mervis and Rosch have similarly characterized basic-level categories as the level at which categories maximize withincategory similarity relative to between-category similarity. The level of abstraction that reflects the basic level, however, may be influenced by both developmental factors and individual knowledge in a given domain (Malt, 1995; Mervis \& Rosch, 1981). Nonetheless, it appears that basic-level categories for many plants and animals may be shared by numerous disparate cultures (Lopez, Atran, Coley, Medin, \& Smith, 1997; Malt, 1995), implying that the basic level may be largely, but not entirely, a product of the structure of the stimuli (Malt, 1995). However, animals with different perceptual systems may perceive that structure differently and, hence, form different categories. Interestingly, the pigeons and monkeys tested by Roberts and Mazmanian failed to discriminate at a level that would be considered the basic level for 
most humans (birds vs. other animals). In the present study, categories were defined a priori and could be considered basic-level categories from the perspective of the experimenters, and the chimpanzees' classification of the stimuli were in agreement with these basic-level categories.

Our chimpanzees (with the exception of the control animal) were previously trained to make samedifferent judgments with different stimuli; however they were never differentially reinforced for discriminating among the type of stimuli used the present experiment. Most comparable studies have involved training on the categories before testing. For example, Irle and Markowitsch (1987) reported discrimination of naturalistic images by squirrel monkeys, without specific categorical training. However, differential reinforcement was used on test trials, and thus it is not possible to determine what contribution the reward contingencies had on the monkeys' overall performance. Hayes and Nissen (1971) reported categorization by a chimpanzee on a sorting task without differential reinforcement. Stimuli included simple objects that could be discriminated on the basis of a small number of discrete characteristics, such as color or size. Their results provided some of the only previous experimental evidence of self-imposed classificatory rules in nonhuman primates.

By measuring viewing times, Fujita and Matsuzawa (1986) reported that one chimpanzee discriminated slides depicting humans from slides with no humans, without training or reinforcement. A number of other studies have similarly measured differential viewing times to demonstrate that several species of macaques can discriminate conspecifics from other macaque species (Fujita, 1987, 1993a, 1993b; Fujita, Watanabe, Widarto, \& Suryobroto, 1997; Swartz, 1982), as well as conspecifics from nonprimate animals (Humphrey, 1974). One advantage of this approach is that it obviates the need for reinforcement from the experimenter. However, it also mandates that the stimuli themselves be intrinsically reinforcing for maintaining the subjects' motivation. In addition, discrete categories are difficult to identify from the data obtained because responses to the stimuli of interest (e.g., humans) were independent of responses to the other stimuli. Thus the procedures used in the present studies may be better suited for inferring categorization, as the animals' responses represented explicit judgments about the relationships between the comparison stimuli.

In general, the present results are similar to the findings of Sands et al. (1982) who reported that rhesus macaques could respond to photographic stimuli in a categorical manner. The monkeys, however, classified some stimuli into categories that would be considered superordinate to the categories reported here. Specifically, the macaques judged humans and monkeys to be the same, whereas our chimpanzee subjects discriminated between chimpanzees and gorillas, thus making a taxonomically finer distinction. In other instances, however, the macaques appeared to make more subordinate distinctions, such as discriminating between blue and red flowers. These differences between macaques and chimpanzees may represent species differences in subjective categorization, context effects due to slightly different testing procedures, or other effects resulting from different rearing conditions.

Wasserman et al. (1988) posited that members of a category resemble one another to a greater extent than they resemble nonmembers. Consequently, stimuli judged to be the same should be perceptually similar, and stimuli judged to be different should be less similar. The discriminations reported here appear to conform to these criteria. The fish and chimpanzee images, for example, were perceptually dissimilar and were consistently classified as different, whereas the different fish were perceptually more similar and were consistently classified as the same. On the other hand, two cross-category comparisons were somewhat perceptually ambiguous, including the cat-tiger and chimp-gorilla comparisons. Tigers are perceptually cat-like, while chimps and gorillas are both apes with black hair and similar body shapes. Consequently, the cat-tiger and chimp-gorilla comparisons were expected to elicit the most inconsistent responses, and the chimpanzees' responses supported this prediction. 
The category of chimpanzee was included in the current study because it was assumed that it would have a unique significance to the subjects. For this reason, we proposed that the chimpanzees would be most discriminative on comparisons involving chimpanzees, although we were unsure whether chimp-chimp comparisons would be judged as same or different. In an earlier study, Humphrey (1974) presented rhesus macaques with pictures of other rhesus macaques and nonprimate animals. Initially, the monkeys appeared to discriminate between individual rhesus macaques, but only between species of nonprimates. After further exposure to pictures of other animals, however, the monkeys began to discriminate between individuals of nonprimate species, thus demonstrating a potential "expertise" effect. In the present study, the chimp-chimp comparison elicited the fewest same responses of all within-category comparisons (see Figure 3), and this pattern was seen with every subject (see Table 2). These findings, while not statistically significant, do allow for the possibility that the chimpanzees were discriminating to a greater extent between individual chimpanzees than between individuals of other species, which might be expected because of their greater familiarity and social exposure to other chimpanzees.

Overall, the current findings suggest that chimpanzees can discriminate among naturalistic stimuli in a manner similar to humans, and that such behavior may emerge independent of specific training. The results appear to satisfy Thomas' (1996) criteria for the use of absolute class concepts, although Premack (1983) criticized conceptual explanations for these kinds of results on the basis that no highly ambiguous exemplars were presented. However, although concepts may not necessarily underlie the current results, the chimpanzees' discriminations were in agreement with experimenter-defined categories. If basic-level categories are determined largely by the structure of the stimuli (Malt, 1995), then it is not surprising that humans and chimpanzees would share many such categories, because it is likely that the perceptual mechanisms that subserve visual processing are quite similar, reflecting the two species' relatively recent evolutionary divergence.

\section{References}

Bhatt, R. S., Wassennan, E. A., Reynolds, W. F., \& Knauss, K. S. (1988). Conceptual behavior in pigeons: Categorization of both familiar and novel examples from four classes of natural and artificial stimuli. Journal of Experimental Psychology: Animal Behavior Processes, 14, 219-234.

Boysen, S. T. (1997). Representation of quantities by apes. Advances in the Study of Behavior, 26, 435462.

Boysen, S. T., Mukobi, K. L., \& Berntson, G. G. (1999). Overcoming response bias using symbolic representations of number by chimpanzees (Pan troglodytes). Animal Learning \& Behavior, 27, 229-235.

Cerella, J. (1979). Visual classes and natural categories in the pigeon. Journal of Experimental Psychology: Human Perception and Performance, 5, 68-77.

D'Amato, M. R., \& Van Sant, P. (1988). The person concept in monkeys (Cebus apella). Journal of Experimental Psychology: Animal Behavior Processes, 14, 43-55.

Eimas, P. D., \& Quinn, P. C. (1994). Studies on the formation of perceptually based basic-level categories in young infants. Child Development, 65, 903-917.

Fabre-Thorpe, M., Richard, G., \& Thorpe, S. J. (1998). Rapid categorization of natural images by rhesus monkeys. NeuroReport, 9, 303-308.

Fujita, K. (1983). Formation of the sameness-difference concept by Japanese monkeys from a small number of color stimuli. Journal of the Experimental Analysis of Behavior, 40, 289-300.

Fujita, K. (1987). Species recognition by five macaque monkeys. Primates, 28, 353-366.

Fujita, K. (1993a). Development of visual preference for closely related species by infant and juvenile macaques with restricted social experience. Primates, 34, 141-150. 
Fujita, K. (1993b). Role of some physical characteristics in species recognition by pigtail monkeys. Primates, 34, 133-140.

Fujita, K., \& Matsuzawa, T. (1986). A new procedure to study the perceptual world of animals with sensory reinforcement: Recognition of humans by a chimpanzee. Primates, 27, 283-291.

Fujita, K., Watanabe, K., Widarto, T. H., \& Suryobroto, B. (1997). Discrimination of macaques by macaques: The case of Sulawesi species. Primates, 38, 233-245.

Hayes, K. J., \& Nissen, C. H. (1971). Higher mental functions of a home raised chimpanzee. In A. M. Schrier \& F. Stolnitz (Eds.), Behavior of nonhuman primates (Vol. 4, pp. 59-115). New York: Academic Press.

Herrnstein, R. J. (1985). Riddles of natural categorization. Philosophical Transactions of the Royal Society of London, B308, 129-144.

Herrnstein, R. J., \& de Villiers, P. A. (1980). Fish as a natural category for people and pigeons. In G. H. Bower (Ed.), The psychology of learning and motivation (Vol. 14, pp. 59-95). New York: Academic Press.

Herrnstein, R. J., \& Loveland, D. H. (1964, October 23). Complex visual concept in the pigeon. Science, 146, 549-551.

Herrnstein, R. J., Loveland, D. H., \& Cable, C. (1976). Natural concepts in pigeons. Journal of Experimental Psychology: Animal Behavior Processes, 2, 285-302.

Himes, G. T. (1999). A chronometric analysis of same/different judgments based on quantity by chimpanzees (Pan troglodytes). Unpublished master's thesis, Ohio State University, Columbus.

Humphrey, N. K. (1974). Species and individuals in the perceptual world of monkeys. Perception, 3, 105114.

Me, E., \& Markowitsch, H. J. (1987). Conceptualization without specific training in squirrel monkeys (Saimiri sciureus): A test using the nonmatch-to-sample procedure. Journal of Comparative Psychology, 101, 305-311.

Lopez, A., Atran, S., Coley, J. D., Medin, D. L., \& Smith, E. E. (1997). The tree of life: Universal and cultural features of folkbiological taxonomies and inductions. Cognitive Psychology, 32, 251-295.

Malt, B. C. (1995). Category coherence in cross-cultural perspective. Cognitive Psychology, 29, 85-148.

Mandler, J. M., \& McDonough, L. (1993). Concept formation in infancy. Cognitive Development, 8, 291318.

Matsuzawa, T. (1990). Spontaneous sorting in human and chimpanzee. In S. T. Parker \& K. R. Gibson (Eds.), "Language" and intelligence in monkeys and apes: Comparative developmental perspectives (pp. 451-468). New York: Cambridge University Press.

Medin, D. L., \& Smith, E. E. (1984). Concepts and concept formation. Annual Review of Psychology, 35, 113-138.

Mervis, C. B., \& Rosen, E. (1981). Categorization of natural objects. Annual Review of Psychology, 32, 89-115.

Neiworth, J. J., \& Wright, A. A. (1994). Monkeys (Macaco mulatto) learn category matching in a nonidentical same-different task. Journal of Experimental Psychology: Animal Behavior Processes, 20, 429-435.

Oakes, L. M., Coppage, D. J., \& Dingel, A. (1997). By land or by sea: The role of perceptual similarity in infants' categorization of animals. Developmental Psychology, 33, 396-407.

Oden, D. L., Thompson, R. K. R., \& Premack, D. (1990). Infant chimpanzees spontaneously perceive both concrete and abstract same/different relations. Child Development, 61, 621-631.

Phillips, K. A. (1996). Natural conceptual behavior in squirrel monkeys (Saimiri sciureus): An experimental investigation. Primates, 37, 327-332.

Premack, D. (1983). Animal cognition. Annual Review of Psychology, 34, 351-362.

Quinn, P. C., Eimas, P. D., \& Rosenkrantz, S. L. (1993). Evidence for representations of perceptually similar natural categories by 3-month-old and 4-month-old infants. Perception, 22, 463-475. 
Roberts, W. A., \& Mazmanian, D. S. (1988). Concept learning at different levels of abstraction by pigeons, monkeys, and people. Journal of Experimental Psychology: Animal Behavior Processes, 14, 4760.

Sands, S. F., Lincoln, C. E., \& Wright, A. A. (1982). Pictorial similarity judgments and the organization of visual memory in the rhesus monkey. Journal of Experimental Psychology: General, 111, 369389.

Schrier, A. M., \& Brady, P. M. (1987). Categorization of natural stimuli by monkeys (Macaco mulatto): Effects of stimulus set size and modification of exemplars. Journal of Experimental Psychology: Animal Behavior Processes, 13, 136-143.

Siegel, S., \& Castellan, N. J. (1988). Nonparametric statistics (2nd ed.). New York: McGraw-Hill.

Spinozzi, G. (1993). Development of spontaneous classificatory behavior in chimpanzees (Pan troglodytes). Journal of Comparative Psychology, 107, 193-200.

Swartz, K. B. (1982). Species discrimination in infant pigtail macaques with pictorial stimuli. Developmental Psychobiology, 16, 219-231.

Tanaka, M. (1995). Object sorting in chimpanzees (Pan troglodytes): Classification based on physical identity, complementarity, and familiarity. Journal of Comparative Psychology, 109, 151-161.

Thomas, R. K. (1996). Investigating cognitive abilities in animals: Unrealized potential. Cognitive Brain Research, 3, 157-166.

Thompson, R. K. R. (1995). Natural and relational concepts in animals. In H. L. Roitblat \& J. A. Meyer (Eds.), Comparative approaches to cognitive science (pp. 175-224). Cambridge, MA: MIT Press.

Thompson, R. K. R., Oden, D. L., \& Boysen, S. T. (1997). Language-naïve chimpanzees (Pan troglodytes) judge relations between relations in a conceptual matching-to-sample task. Journal of Experimental Psychology: Animal Behavior Processes, 23, 31-43.

Wasserman, E. A., Kiedinger, R. E., \& Bhatt, R. S. (1988). Conceptual behavior in pigeons: Categories, subcategories, and pseudocategories. Journal of Experimental Psychology: Animal Behavior Processes, 14, 235-246.

Yoshikubo, S. (1985). Species discrimination and concept formation by rhesus monkeys (Macaco mulatto). Primates, 26, 285-299.

Zayan, R., \& Vauclair, J. (1998). Categories as paradigms for comparative cognition. Behavioural Processes, 42, 87-99. 